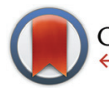

CrossMark \& click for updates

Cite this: Org. Biomol. Chem., 2015, 13, 11414

Received 26th September 2015, Accepted 13th October 2015

DOI: $10.1039 / c 50 b 02009 c$

www.rsc.org/obc

\section{Chemical chain termination resolves the timing of ketoreduction in a partially reducing iterative type I polyketide synthase $\uparrow$}

\author{
Hirokazu Kage, ${ }^{a}$ Elena Riva, ${ }^{b}$ James S. Parascandolo, ${ }^{b}$ Martin F. Kreutzer, ${ }^{a}$ \\ Manuela Tosin*b and Markus Nett*a
}

\begin{abstract}
Synthetic chain terminators were used to capture the biosynthetic intermediates from a partially reducing iterative type I polyketide synthase, which is integrated into a multimodular biosynthesis enzyme. The off-loaded metabolites clarified the timing of ketoreduction and aromatization in the assembly of the antibiotic micacocidin.
\end{abstract}

Polyketides represent an important class of secondary metabolites that are found in bacteria, fungi, plants and marine organisms alike. ${ }^{1}$ Their structural diversity arises from the action of large multifunctional proteins. The polyketide synthases (PKSs) select the acyl-CoA building blocks for incorporation and they control the number of chain extensions, the reductive processing and cyclization pattern. ${ }^{2}$ Depending on their mode of action, that is, whether or not they catalyse multiple elongation steps, PKSs are categorized as iterative or noniterative. ${ }^{3}$ While the programming of noniterative PKSs is often predictable, ${ }^{4}$ the mechanistic rules of iterative PKSs remain to be fully understood. ${ }^{5}$ This is particularly true for partially reducing (PR)-PKSs, which introduce hydroxyl groups at specific sites of the growing polyketide chain and, thereby, set the structural basis for subsequent cyclization reactions. In general, PR-PKSs share a highly conserved domain architecture, which distinguishes them from non-reducing (NR) and highly reducing (HR) PKSs. They typically possess a ketoreductase (KR) domain, but they lack dehydratase (DH) or enoylreductase (ER) domains for further reductive processing. PR-PKSs are mostly encountered as discrete proteins, catalysing the production of small aromatic molecules (Table 1). Examples include 6-methylsalicylic acid synthase (6-MSAS), which is quite common in fungi ${ }^{6}$ but can also be found in several bacteria, ${ }^{7} \mathrm{NcsB}$ and AziB, which provide the bio-

\footnotetext{
${ }^{a}$ Leibniz Institute for Natural Product Research and Infection Biology, Hans-Knöll-Institute, Adolf-Reichwein-Str. 23, D-07745 Jena, Germany. E-mail: markus.nett@hki-jena.de; Fax:+49 3641 5320811; Tel: +49 36415321297 ${ }^{b}$ Department of Chemistry, University of Warwick, Library Road, Coventry CV4 7AC, UK. E-mail:m.tosin@warwick.ac.uk; Tel: +44 (0)2476572878

$\dagger$ Electronic supplementary information (ESI) available. See DOI: 10.1039/ c5ob02009c
}

Table 1 Overview of PR-PKSs, their domain organisation and products

Protein Domain architecture

synthetic building blocks 2-hydroxyl-5-methyl-naphthoic acid and 5-methyl-naphthoic acid, ${ }^{8,9}$ as well as the mellein synthase SACE5532. ${ }^{10}$

Recently, we identified a PR-PKS as a constituent of the multimodular enzyme MicC in the biosynthetic pathway to the antibiotic micacocidin. ${ }^{11}$ In vitro studies confirmed that the PR-PKS is loaded with hexanoic acid via intraprotein transfer from an N-terminal acyl carrier protein (ACP) domain prior to iterative chain elongation. ${ }^{12,13}$ Although the described priming mechanism appears to be quite rare for PR-PKSs, it is commonly observed in fungal NR-PKSs. ${ }^{14}$ Another deviation from the prototypical PR-PKS is the lack of a thioester hydrolase (TH) domain in MicC. In 6-MSAS biosynthesis, the TH domain was shown to mediate the final product release, ${ }^{15}$ and the same function is also conceivable for NcsB, AziB and 
SACE5532. In micacocidin biosynthesis, however, the assumed product of the PR-PKS reaction (i.e., 6-pentylsalicylic acid) is immediately transferred to a non-ribosomal peptide synthetase (NRPS) module. A premature offload by a TH domain is thus not required. Functional interrogation of the micacocidin PR-PKS in a heterologous host already confirmed that the integrated KR domain is indispensable for the correct assembly of 6-pentylsalicylic acid. ${ }^{12}$ Due to the peculiarities of MicC in comparison with other PR-PKSs, several questions concerning its function have remained unsolved. In particular, the timing of the regioselective ketoreduction as well as the cyclization and aromatization of the linear tetraketide precursor were not clear. To achieve a better understanding of the programming of a PR-PKS, which is embedded in a multimodular enzyme, we sought to elucidate the sequence of the aforementioned reactions.

Initially, the active site of the KR domain in MicC was inactivated by site-directed mutagenesis. The mutation was introduced into the chromosome of the native micacocidin producer Ralstonia solanacearum GMI1000 by homologous recombination. The resulting mutant strain RS36 was incapable of synthesizing the antibiotic (1), but accumulated a metabolite (2) that was only present at low levels in the wild type strain (Fig. 1). After purifying this compound by HPLC, 2 could be identified as 4-methoxy-6-pentyl-2H-pyran-2-one through comparison of its NMR and mass spectroscopic data with literature values (Fig. $\mathrm{S} 1$ and $\mathrm{S} 2 \dagger) .{ }^{16}$ Biosynthetically, 2 is proposed to originate from hexanoic acid, which would be extended by two decarboxylative Claisen condensations with malonyl-CoA and, eventually, undergo a lactonization reaction. A disruption of the ketosynthase (KS) domain in MicC abolished the production of 2 and, thereby, confirmed the pro-
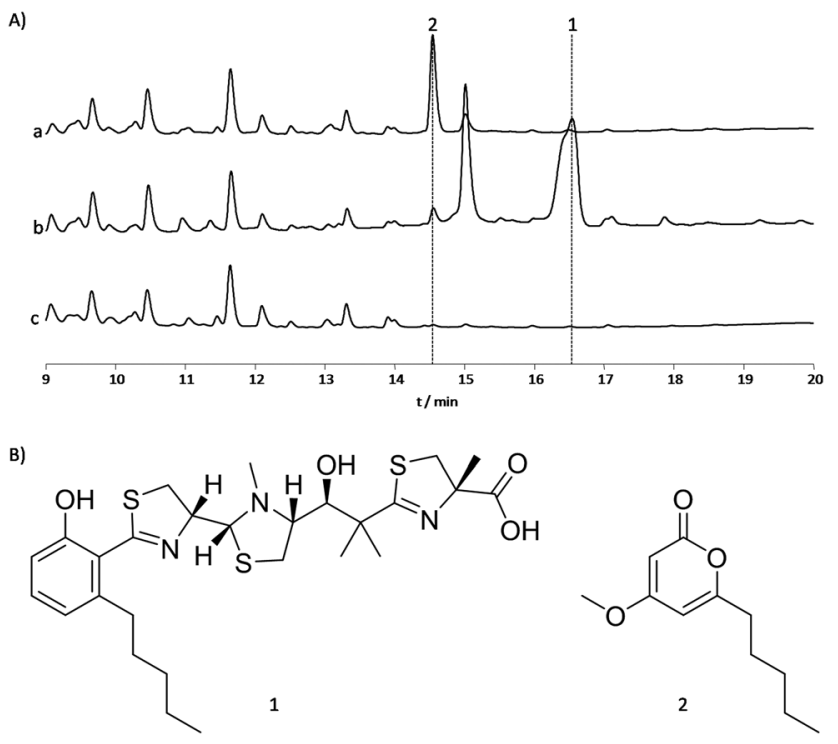

Fig. 1 Metabolic profiles of crude culture extracts from the $R$. solanacearum strains RS36 (profile a, MicC-KR mutant), GMI1000 (profile b, wild type), and RS31 (profile $\mathrm{C}, \Delta$ micC mutant) (A). Chemical structures of the metabolites 1 and 2 (B). posed biosynthetic relationship (Fig. 1A, profile c). Despite thorough analysis of the extract from strain RS36 by LC/MS, only traces of putative tetraketide products were detected. This suggests that the ketoreduction of the intact micacocidin PR-PKS takes place at the stage of the triketide intermediate. If this step is omitted, e.g., due to an impaired KR domain, the assembly line-based biosynthesis would likely be aborted and lead to the occurrence of 2 .

To get further insights into the sequence of biosynthetic reactions and to verify the proposed timing of ketoreduction, we decided to intercept the enzymatic assembly of 6-pentylsalicylic acid. Chemical probes, such as malonyl carba(dethia) pantetheines and $\mathrm{N}$-acetylcysteamines have been shown to compete with acyl carrier protein (ACP)-bound substrates for polyketide chain elongation and to induce a premature release of truncated intermediates. Since the resulting products cannot be reloaded onto the assembly line, they become available for LC/MS characterization. ${ }^{17}$ It should be noted that the aforementioned probes have been successfully utilised both for in vitro ${ }^{17}$ and in vivo ${ }^{18-21}$ investigations of modular PKSs.

In the present study, $R$. solanacearum GMI1000 was initially grown in the presence of malonyl carba(dethia)- $N$-acetyl cysteamine methyl ester 3a, which hydrolyses in vivo to generate the active probe $6 a^{18-20}$ Following a 3-day cultivation, the bacterial cultures were extracted with ethyl acetate to recover the offloaded intermediates. LC/HRMS analysis revealed the presence of molecular species, which were not present in control samples and could be assigned to putative diketide and tetraketide intermediates (Fig. 2A and S3-S7†). The effective occurrence of these molecules was verified in parallel experiments in which $R$. solanacearum cultures were supplemented with the deuterated probe $\mathbf{3 b}$ in order to introduce a distinctive mass shift in the corresponding species. Feeding experiments with the $N$-pentynoyl probe 4 led to analogous results and the corresponding diketide and tetraketide intermediates were also subjected to mass fragmentation studies, which support their putative structure (Fig. S8-S12 $\dagger$ ). Although feeding experiments with probes $\mathbf{3 a}, \mathbf{3} \mathbf{b}$ and $\mathbf{4}$ were repeated several times with varying concentrations of the synthetic chain terminator, no masses corresponding to triketide species were detected and the precise timing of the ketoreduction remained unclear. Assuming that cellular uptake and/or in vivo hydrolysis of the fed methyl esters might be limiting factors for the detection of all biosynthetic intermediates, ${ }^{18,19}$ we resorted to the use of malonyl carba(dethia)- $N$-decanoyl cysteamine methyl ester (5), which has been found to be more efficiently hydrolysed in vivo to its corresponding carboxylate $8 .^{21} \mathrm{LC} / \mathrm{MS}$ analyses of $R$. solanacearum extracts grown in the presence of $\mathbf{5}$ revealed the capture of the same diketide and tetraketide species observed in the previous feeding experiments. More importantly, the masses of putative triketide species were also detected (Fig. 2B and ESI $\dagger$ ). MS/MS analysis of the putative reduced triketide yielded diagnostic peaks at $\mathrm{m} / \mathrm{z} 226$ and 208, which correspond to the formation of a protonated cyclic imine following loss of the decanoyl chain during fragmentation and subsequent dehydration. ${ }^{21}$ This confirmed the 

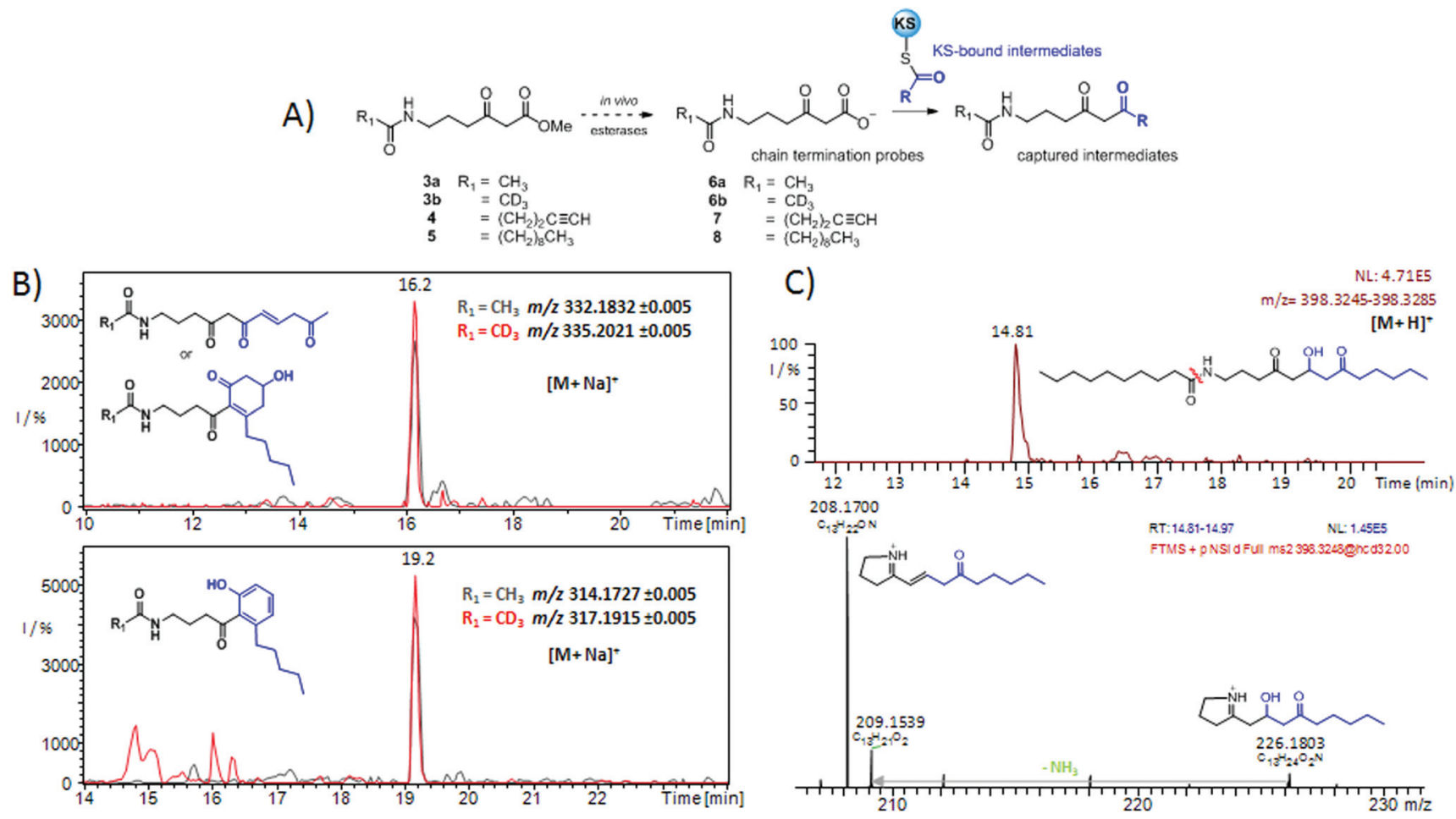

Fig. 2 Chain termination probes 6-8 utilised for the capture of polyketide intermediates in micacocidin assembly (A). Extracted ion chromatograms for putative tetraketides (sodiated addicts) captured from $R$. solanacearum GMI1000 cultures grown in the presence of the methyl esters $3 a$ and $3 \mathrm{~b}$, which hydrolyse to $6 \mathrm{a}$ and $6 \mathrm{~b}(\mathrm{~B}) .{ }^{18-21}$ Extracted ion chromatogram and HR-MS ${ }^{2}$ analyses for the protonated adduct of a putative reduced triketide identified from the growth of $R$. solanacearum GMI1000 in the presence of the $N$-decanoyl probe 8 (C). ${ }^{21}$

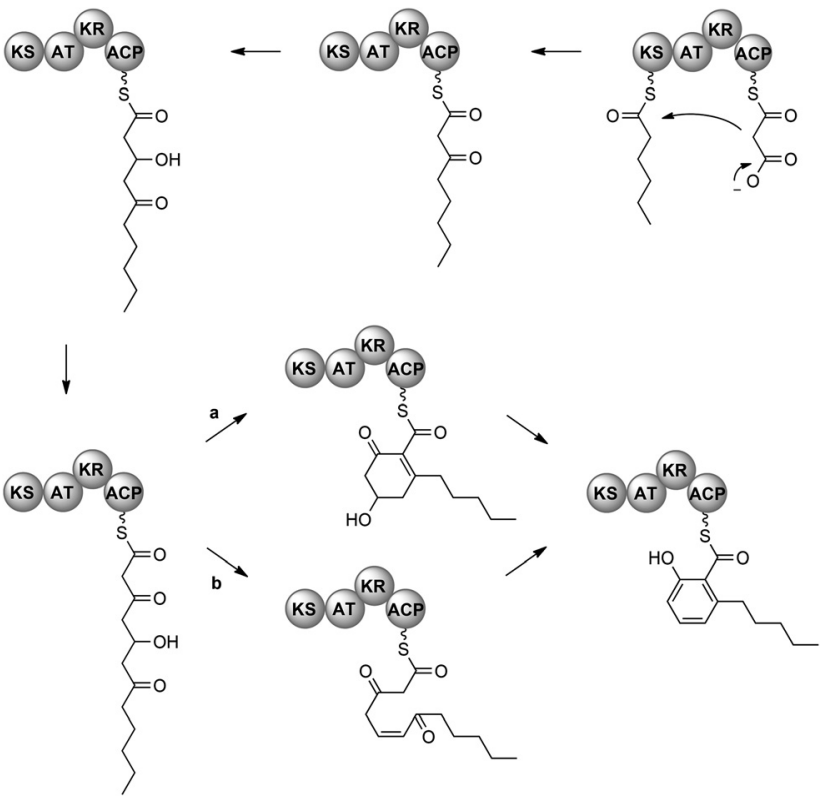

Fig. 3 Model for the assembly of enzyme-bound 6-pentylsalicylate by the PR-PKS from micacocidin biosynthesis.

identity of the hydroxylated triketide species and established the timing of the ketoreduction during the MicC-mediated assembly of 6-pentylsalicylic acid (Fig. 3).
The selective $\beta$-ketoreduction would hence take place immediately after the second Claisen condensation with malonyl-CoA. Because no distinct masses of unsaturated triketides were observed during the different chain termination experiments, we assume that the intermediary hydroxyl group is not eliminated before the final chain elongation. Subsequently, an elongated 5-hydroxy tetraketide must undergo an intramolecular aldol condensation and a likely spontaneous dehydration, with enolisation eventually giving rise to enzymebound 6-pentylsalicylate. These experiments also confirm that MicC encodes for the minimum set of proteins necessary to generate 6-alkyl salicylic acid derivatives. The mechanism of the 6-pentylsalicylate moiety transfer to the MicC downstream domains and others is currently under investigation.

\section{Conclusions}

In the present study, chemical probes were used to elucidate the programming of a PR-PKS which, unlike other related enzymes, is integrated into a multimodular assembly line. Notwithstanding this specific feature, our results show that the investigated PR-PKS follows the same logic as the previously characterized 6-MSAS, ${ }^{15,22}$ thereby establishing a unified paradigm for the biosynthetic mechanism of these proteins. Moreover, we demonstrate that chemical chain termination is a useful tool for the functional interrogation of iterative PKSs 
under in vivo conditions. The possibility to trap an entire range of biosynthetic intermediates in simple feeding experiments with nonhydrolysable malonyl ACP analogues complements existing methods for the analysis of these enzymes. Since selective domain inactivations can abort the biosynthesis at a specific step, the functional study of iterative PKSs usually involves domain swaps, ${ }^{22,23}$ heterologous reconstitution, ${ }^{10 a, 24}$ or the synthesis of highly reactive substrates for in vitro tests. ${ }^{10 b}$ When compared to these methods, the advantage of chemical chain termination clearly lies in its convenience.

\section{Acknowledgements}

The idea for this project was born on occasion of the $3^{\text {rd }}$ Transatlantic Frontiers of Chemistry Symposium, which was organised by the American Chemical Society (ACS), the Gesellschaft Deutscher Chemiker (GDCh), and the Royal Society of Chemistry (RSC). The authors gratefully acknowledge Andrea Perner (Hans-Knöll-Institute Jena) and Dr Lijiang Song (Warwick Chemistry) for initial LC/HRMS measurements. Financial support was provided by the Leibniz Association, BBSRC (project grant BB/J007250/1 to M. T.), the Institute of Advanced Studies (IAS) at Warwick (Postdoctoral Fellowship to E. R.) and EPSRC (DTA studentship to J. S. P.)

\section{Notes and references}

1 (a) D. O'Hagan, The Polyketide Metabolites, 1999, Ellis Horwood, Chichester; (b) K. J. Weissman, Methods Enzymol., 2009, 459, 3.

2 C. Hertweck, Angew. Chem., Int. Ed., 2009, 48, 4688.

3 R. J. Cox, Org. Biomol. Chem., 2007, 5, 2010.

4 M. Nett, Prog. Chem. Org. Nat. Prod., 2014, 99, 199.

5 K. M. Fisch, RSC Adv., 2013, 3, 18228.

6 (a) J. Beck, S. Ripka, A. Siegner, E. Schiltz and E. Schweizer, Eur. J. Biochem., 1990, 192, 487-498; (b) I. Fujii, Y. Ono, H. Tada, K. Gomi, Y. Ebizuka and U. Sankawa, Mol. Gen. Genet., 1996, 253, 1; (c) P. Lu, A. Zhang, L. M. Dennis, A. M. Dahl-Roshak, Y.-Q. Xia, B. Arison, Z. An and J. S. Tkacz, Mol. Genet. Genomics, 2005, 273, 207.

7 (a) S. Gaisser, A. Trefzer, S. Stockert, A. Kirschning and A. Bechthold, J. Bacteriol., 1997, 179, 6271; (b) W. Ding, C. Lei, Q. He, Q. Zhang, Y. Bi and W. Liu, Chem. Biol., 2010, 17, 495; (c) S. G. Van Lanen, T. J. Oh, W. Liu, E. Wendt-Pienkowski and B. Shen, J. Am. Chem. Soc., 2007, 129, 13082; (d) W. L. Lu, N. Roongsawang and T. Mahmud, Chem. Biol., 2011, 18, 425.

8 B. Sthapit, T. J. Oh, R. Lamichhane, K. Liou, H. C. Lee, C. G. Kim and J. K. Sohng, FEBS Lett., 2004, 566, 201.

9 Q. F. Zhao, Q. L. He, W. Ding, M. C. Tang, Q. J. Kang, Y. Yu, W. Deng, Q. Zhang, J. Fang, G. L. Tang and W. Liu, Chem. Biol., 2008, 15, 693.
10 (a) H. Sun, C. L. Ho, F. Ding, I. Soehano, X.-W. Liu and Z.-X. Liang, J. Am. Chem. Soc., 2012, 134, 11924; (b) I. Soehano, L. Yang, F. Ding, H. Sun, Z. J. Low, X. Liu and Z.-X. Liang, Org. Biomol. Chem., 2014, 12, 8542.

11 M. F. Kreutzer, H. Kage, P. Gebhardt, B. Wackler, H. P. Saluz, D. Hoffmeister and M. Nett, Appl. Environ. Microbiol., 2011, 77, 6117.

12 H. Kage, M. F. Kreutzer, B. Wackler, D. Hoffmeister and M. Nett, Chem. Biol., 2013, 20, 764.

13 M. F. Kreutzer, H. Kage, J. Herrmann, J. Pauly, R. Hermenau, R. Müller, D. Hoffmeister and M. Nett, Org. Biomol. Chem., 2014, 12, 113.

14 (a) J. M. Crawford, B. C. R. Dancy, E. A. Hill, D. W. Udwary and C. A. Townsend, Proc. Natl. Acad. Sci. U. S. A., 2006, 103, 16728; (b) H. Zhou, J. Zhan, K. Watanabe, X. Xie and Y. Tang, Proc. Natl. Acad. Sci. U. S. A., 2006, 105, 6249; (c) A. Al Fahad, A. Abood, K. M. Fisch, A. Osipow, J. Davison, M. Avramović, C. P. Butts, J. Piel, T. J. Simpson and R. J. Cox, Chem. Sci., 2014, 5, 523.

15 T. Moriguchi, Y. Kezuka, T. Nonaka, Y. Ebizuka and I. Fujii, J. Biol. Chem., 2010, 285, 15637.

16 A. Evidente, M. C. Zonno, A. Andolfi, C. Troise, A. Cimmino and M. Vurro, J. Antibiot., 2012, 65, 203.

17 M. Tosin, L. Betancor, E. Stephens, W. M. A. Li, J. B. Spencer and P. F. Leadlay, ChemBioChem, 2010, 11, 539.

18 M. Tosin, Y. Demydchuk, J. S. Parascandolo, C. Blasco Per, F. J. Leeper and P. F. Leadlay, Chem. Commun., 2011, 47, 3460 .

19 M. Tosin, L. Smith and P. F. Leadlay, Angew. Chem., Int. Ed., 2011, 50, 11930.

20 J. Franke, K. Ishida and C. Hertweck, Angew. Chem., Int. Ed., 2012, 51, 11611.

21 E. Riva, I. Wilkening, S. Gazzola, W. M. A. Li, L. Smith, P. F. Leadlay and M. Tosin, Angew. Chem., Int. Ed., 2014, 53, 11944.

22 T. Moriguchi, Y. Ebizuka and I. Fujii, ChemBioChem, 2008, 9, 1207.

23 K. M. Fisch, W. Bakeer, A. A. Yakasai, Z. Song, J. Pedrick, Z. Wasil, A. M. Bailey, C. M. Lazarus, T. J. Simpson and R. J. Cox, J. Am. Chem. Soc., 2011, 133, 16635.

24 (a) J. Antosch, F. Schaefers and T. A. M. Gulder, Angew. Chem., Int. Ed., 2014, 53, 3011; (b) Y. Li, H. Chen, Y. Xie, H. Wang, R. L. Cerny, Y. Shen and L. Du, Angew. Chem., Int. Ed., 2014, 53, 7524; (c) B. Busch, N. Ueberschaar, S. Behnken, Y. Sugimoto, M. Werneburg, N. Traitcheva, J. He and C. Hertweck, Angew. Chem., Int. Ed., 2013, 52, 5285; (d) B. Busch, N. Ueberschaar, Y. Sugimoto and C. Hertweck, J. Am. Chem. Soc., 2012, 134, 12382; (e) J. M. Winter, M. Sato, S. Sugimoto, G. Chiou, N. K. Garg, Y. Tang and K. Watanabe, J. Am. Chem. Soc., 2012, 134, 17900; $(f)$ A. O. Zabala, Y.-H. Chooi, M. S. Choi, H.-C. Lin and Y. Tang, ACS Chem. Biol., 2014, 9, 1576; (g) Y.-H. Chooi, C. Krill, R. A. Barrow, S. Chen, R. Trengove, R. P. Oliver and P. S. Solomon, Appl. Environ. Microbiol., 2015, 81, 177. 